\title{
Tsafon
}

Revue d'études juives du Nord

$74 \mid 2017$

La Déclaration Balfour, vers l'État d'Israël ?

\section{L'Alliance israélite universelle et le sionisme (1914-1919)}

Catherine Nicault

\section{OpenEdition}

1 Journals

Édition électronique

URL : https://journals.openedition.org/tsafon/398

DOI : $10.4000 /$ tsafon.398

ISSN : 2609-6420

Éditeur

Association Jean-Marie Delmaire

Édition imprimée

Date de publication : 1 décembre 2017

Pagination : 49-64

ISSN : 1149-6630

\section{Référence électronique}

Catherine Nicault, «L'Alliance israélite universelle et le sionisme (1914-1919) », Tsafon [En ligne], 74 |

2017, mis en ligne le 31 mai 2018, consulté le 23 juin 2021. URL : http://journals.openedition.org/ tsafon/398; DOl : https://doi.org/10.4000/tsafon.398

Tsafon. Revues d'études juives du Nord 


\title{
L'Alliance israélite universelle et le sionisme (1914-1919)
}

\author{
Catherine Nicault
}

Le sionisme politique herzlien fut combattu dès sa naissance par l'Alliance israélite universelle (AIU) ou plutôt ses dirigeants, essentiellement le président Narcisse Leven (1898-1915), le viceprésident Arnold Netter, président intérimaire de 1915 à 1920, et le secrétaire Jacques Bigart (depuis 1892) ${ }^{1}$. Non que l'Alliance, en tout cas son état-major, se désintéresse de la Palestine : la création de l'école d'agriculture de Mikveh Israël en 1870 ainsi que celle d'un réseau scolaire en Eretz Israel entre les années 1870 et 1900 montrent le contraire $^{2}$. Mais, l'institution se donne avant tout pour mission de diffuser auprès des Juifs d'Orient le modèle français d'émancipation et d'assimilation. Il s'ensuit un fossé idéologique profond entre l'AIU et

\footnotetext{
*Université de Reims.

Une première version de cet article, directement inspirée du chapitre 5 («Face au sionisme ») rédigé par l'auteure pour André Kaspi (dir.), Histoire de l'Alliance israélite universelle. De 1860 à nos jours, Paris, Armand Colin, 2010, pp. 189-226 (désormais : C. Nicault, «Face au sionisme »), a été présentée lors du colloque organisé en octobre 2010 par l'Institut Ben Tsvi à Jérusalem pour le $150^{\mathrm{e}}$ anniversaire de 1'AIU.

${ }^{1}$ Sur cette question : C. Nicault, «Face au sionisme»; idem, La France et le sionisme (1897-1948): Une rencontre manquée?, Paris, Éditions Calmann-Lévy, collection « Diaspora », 1992 ; Michel Abitbol, Les deux Terres promises : les Juifs de France et le sionisme (1897-1945), Paris, Perrin, coll. «Tempus », 2010 [1 ${ }^{\text {ère }}$ éd. Olivier Orban 1989].

${ }^{2}$ L'AIU inaugure son réseau scolaire en Palestine par l'ouverture d'une école de garçons à Jaffa qui eut une existence brève et passablement chaotique (1864-1873). À Mikveh Israel (1870), à l'école de garçons de Haïfa (fin 1881) et à l'École professionnelle de garçons de Jérusalem (1882), des créations cette fois durables, s'ajoutèrent dans les années 1890 et 1900 une dizaine d'établissements à Acre, Haïfa, Jérusalem, Safed, Jaffa et Tibériade. Georges Weill, Émancipation et progrès. L'Alliance israélite universelle et les droits de l'Homme, Paris, Les Éditions du Nadir, 2000, (Annexe : Liste des écoles).
} 
l'Organisation sioniste mondiale, fondée en 1897, et une vive rivalité nés de leurs deux visions irréconciliables de l'avenir des Juifs. À cet antagonisme de fond s'ajoute la conviction chez les hommes de l'Alliance que le projet sioniste est irréalisable sur le plan pratique, et nocif tant pour les colonies palestiniennes « Rothschild» que pour le statut des sujets juifs du Sultan et celui des «Israélites » émancipés. Enfin, ce conflit est un conflit d'appareils et de praxis politiques entre une société de notables qui privilégient l'intercession et la discrétion, et une organisation de masse qui recherche, au contraire, la lumière et la plus grande visibilité possible sur la scène publique.

Dès avant la Grande Guerre, diverses escarmouches ont poussé l'AIU, déjà placée sur la défensive, à chercher appui auprès du Quai d'Orsay, qui apprécie en elle un instrument performant de diffusion de la culture et de l'influence française contre le sionisme réputé «allemand $»^{3}$. Pour autant, l'institution entendait éviter toute polémique publique avec la nouvelle «doctrine» nationale juive, considérant qu'elle s'éteindrait d'elle-même. Si bien que, lorsque la guerre éclate en Europe en août 1914, les hommes de l'Alliance sont certains que la dernière heure du mouvement sioniste a sonné. Or, contrairement à leurs prévisions, celui-ci non seulement survit mais remporte une victoire décisive en novembre 1917 sur l'Alliance et le projet émancipateur qu'elle incarne en obtenant des Britanniques, qui s'apprêtent alors à occuper la Palestine ottomane, la Déclaration Balfour, base du futur Foyer national juif.

Malgré l'obstacle de lacunes documentaires considérables ${ }^{4}$, nous nous proposons d'approfondir cette défaite de l'Alliance à la lumière de diverses recherches entreprises pour notre thèse, consacrée à l'échec des autorités françaises à s'adjuger la Palestine à la faveur de la guerre, et surtout après celle-ci ${ }^{5}$. On connaît l'adage voulant que l'histoire soit écrite par les vainqueurs. Nous espérons montrer encore que l'histoire des vaincus est tout aussi riche d'enseignements.

\footnotetext{
${ }^{3} \mathrm{Au}$ tournant du siècle, Herzl et ses partisans ont tenté, en vain, de «noyauter » le Comité central de l'Alliance. À la même époque, commence en divers lieux une campagne des sionistes contre l'enseignement «antinational» que délivrerait, selon eux, l'Alliance, laquelle aboutit à l'éviction quasiment totale de cette dernière de Bulgarie. Cf. C. Nicault, La France et le sionisme..., op. cit., pp. 46-47 et «Face au sionisme », notamment pp. 92-93.

${ }^{4}$ En ce qui concerne en particulier l'action menée par le baron Edmond de Rothschild, pour laquelle il ne semble pas exister d'archives directes, tandis que les archives de l'Alliance israélite universelle sont étonnamment pauvres à ce sujet.

${ }^{5}$ Dans La France et le sionisme..., op. cit.
} 


\section{Du sort des Juifs russes à la question sioniste : l'entrée en scène d'une « diplomatie israélite alliée » (1914-1915)}

L'une des raisons des difficultés auxquelles se heurte l'historien désireux d'analyser cette bataille contre le sionisme est que l'Alliance ne l'a pas livrée seule ni en son nom propre, du moins dans les premières années de la guerre. Dans les premiers mois du conflit entre l'Entente (France, Grande-Bretagne, Russie) et les Puissances centrales (Allemagne, Autriche-Hongrie, Turquie), plusieurs organisations du judaïsme occidental, porteuses depuis le siècle précédent du projet émancipateur, unissent en effet leurs efforts pour tenter d'arracher, à la faveur du conflit, une solution à la question lancinante qui occupe depuis les années 1880 l'élite des Juifs occidentaux : le sort du judaïsme russe, déjà persécuté dans l'Empire tsariste en temps de paix et désormais directement exposé sur le front oriental aux soupçons de trahison et aux dévastations de la guerre ${ }^{6}$.

De leur côté, les chancelleries française et britannique s'inquiètent des sentiments pro-allemands que, leur rapportent leurs ambassades, nourriraient aux États-Unis les Juifs d'origine allemande et surtout ceux originaires de Russie, de loin majoritaires. En fait, l'opinion juive américaine n'est pas réellement pro-allemande; elle est avant tout antirusse, ce qui n'est pas la même chose. Reste qu'au Quai d'Orsay et au Foreign Office, on redoute qu'elle ne fasse obstacle à une éventuelle entrée en guerre des États-Unis aux côtés de l'Entente et, dans l'immédiat surtout, à l'octroi de crédits aux Alliés français, britanniques et russes par le système bancaire américain, censé être dominé par les Juifs.

Ces spéculations inspirent aux hommes de l'Alliance l'idée qu'il serait possible d'obtenir des autorités françaises et britanniques qu'elles fassent pression sur leur allié russe pour qu'il traite mieux ses sujets juifs, en leur assurant que cette initiative ferait remonter la cote de l'Entente en Amérique. C'est sur cet agenda - priorité donnée à l'objectif russe - et

\footnotetext{
${ }^{6}$ Marc Levene, War, Jews, and the New Europe. The diplomacy of Lucien Wolf, 19141919, The Litman Library of Jewish Civilization, Oxford University Press, 1992. Depuis 1881, les espoirs d'une émancipation légale des Juifs russes par le pouvoir tsariste ont fait long feu. De plus, depuis la conclusion de l'alliance franco-russe au début des années 1890 et celle de l'« entente » anglo-russe en 1907, les organisations de notables juifs français et britanniques s'étaient faites très discrètes sur la question, ne voulant pas gêner la politique extérieure de leurs gouvernements.
} 
sur cette stratégie que se constitue, entre novembre 1914 et le printemps 1915, un petit groupe de pression qui entend être le fer de lance d'une sorte de «diplomatie israélite » à l'échelle de l'Entente, dont l'historien Mark Levene a proposé une première approche du côté britannique ${ }^{7} \mathrm{Au}$ centre de ce groupe se trouvent l'AIU et le Jewish Conjoint Committee dont le directeur Lucien Wolf est chargé de piloter l'action politique commune $^{8}$, ainsi que le baron Edmond de Rothschild, dont l'influence, pour être certaine, n'est pas toujours facile à cerner avec précision. Autour de ce noyau décisionnel majeur gravitent des organisations « sœurs » moins impliquées (l'American Jewish Committee), à Rome (le Conserzio delle Comunità Isralitiche Italiane), à Bucarest (L'Union des Juifs indigènes) et à Petrograd (le Bureau politique).

Or, les premiers efforts déployés en ce sens par Lucien Wolf, leur porte-parole, montrent que les autorités de Paris et de Londres sont loin de vouloir courir le risque de fâcher leur indispensable allié russe en s'ingérant dans ses affaires intérieures. Prenant conscience que les temps ne sont pas mûrs, Lucien Wolf et ses commanditaires confèrent alors à leur «diplomatie israélite» un autre objectif: battre sur leur propre terrain les sionistes qui, malgré la désorganisation interne induite par la situation de guerre, aspirent à tirer parti de l'engagement de l'Empire ottoman dans le camp des puissances centrales pour faire avancer la cause nationale juive ${ }^{9}$. On constate en effet qu'à la fin de février ou au début de mars 1915, Wolf inscrit pour la première fois un objectif sionisant sur l'agenda de son action politique : «Demander des facilités particulières pour l'immigration et la colonisation juives et pour le libre développement des institutions juives et de la vie culturelle juive en Palestine $\gg{ }^{10}$. Pourquoi cette initiative, et comment expliquer en particulier que l'Alliance, qui suit de très près les démarches de Lucien Wolf, ait accepté de le suivre sur ce terrain ?

Il se peut qu'en Grande-Bretagne comme en France, les rumeurs qui courent très tôt dans les milieux juifs sur des contacts entre Weizmann et des officiels britanniques - lesquels, en réalité, sont

\footnotetext{
${ }^{7}$ Mark Levene, op. cit.

${ }^{8}$ Le Jewish Conjoint Committee est, depuis 1878, l'instrument commun de politique étrangère de deux grandes organisations historiques du judaïsme britannique, le Board of Deputies of British Jews et l'Anglo-Jewish Association.

${ }^{9}$ David Vital, Zionism : The Crucial Phase, Oxford, Clarendon Press, 1987.

${ }^{10}$ Archives de l'Alliance israélite universelle (désormais AAIU), lettre à Jacques Bigart citée par M. Levene, op. cit., p. 79, et traduite par nos soins. Wolf écrit dans les mêmes termes à Cyrus Adler de l'American Jewish Committee en septembre 1915.
} 
négligeables et n'engagent encore personne - aient poussé Edmond de Rothschild, les dirigeants de l'AIU, Lucien Wolf et ses commanditaires britanniques à prendre cette initiative dans l'intention de saboter des avancées sionistes éventuelles. Cependant, Marc Levene prête à Lucien Wolf un motif plus subtil qui nous semble convaincant au vu de sa conduite ultérieure. Selon l'historien britannique, soulever la question des droits des Juifs en Palestine serait apparu au porte-parole des intérêts israélites le meilleur moyen de garder ouvert avec le Foreign Office, et secondairement avec le Quai d'Orsay, un dialogue compromis par leur refus de peser sur le gouvernement russe en faveur de ses sujets juifs, mais qu'il reste indispensable de maintenir pour pouvoir reprendre, le moment venu, les négociations relatives aux Juifs russes. Au demeurant, bien que résolument hostile au " postulat national », Wolf a été et reste sensible à la palestinophilie non politique d'une partie du mouvement des Amants de Sion.

Reste à comprendre d'où sort cette idée surprenante. Parmi bien des hypothèses plausibles et, du reste, nullement exclusives les unes des autres, nous sommes tentée de l'attribuer à Edmond de Rothschild. Élisabeth Antébi a établi, en effet, que le Baron ambitionnait alors de fédérer autour de lui le gros des forces nationales juives ${ }^{11}$. Le «Bienfaiteur» des colonies palestiniennes est toujours très opposé au sionisme politique herzlien, mais il a interprété la victoire au congrès de 1911 des sionistes pratiques, favorables au développement de la colonisation juive en Palestine même sans la garantie d'une charte des puissances, comme une revanche personnelle sur Herzl. Depuis lors, il se plaît à se dire lui-même "sioniste", même si ses idées en matière palestinienne ne vont pas au-delà de l'obtention d'un statut d'autonomie pour le Yishouv (la communauté juive) et les institutions juives d'Eretz Israel, et s'il exclut la création, pour l'instant en tout cas, d'une entité politique qui risquerait, selon lui, de provoquer une levée de boucliers chez les chrétiens et la colère des Arabes. Pour protéger les destinées de cette future autonomie juive, il prône par ailleurs une tutelle britannique et non française, tant il redoute l'influence des milieux catholiques sur la politique que mènerait Paris en Palestine. C'est notamment cette préférence pour une tutelle britannique qui explique les excellentes relations, par ailleurs fondées sur un malentendu fondamental sur la

\footnotetext{
${ }^{11}$ Élizabeth Antébi, Edmond de Rothschild. L'homme qui racheta la Terre sainte, Paris, Éditions du Rocher, 2003. Précisons que le rôle du Baron dans les grandes manœuvres politiques que nous analysons ici ont échappé à l'auteure du livre.
} 
nature du projet sioniste, qu'ont nouées le Baron et Chaïm Weizmann un peu avant la guerre ${ }^{12}$.

Outre le fait que ces éléments de contexte semblent peser fortement en faveur de la thèse selon laquelle le Baron serait le père de l'idée d'utiliser le facteur «sioniste» aux fins propres de la «diplomatie israélite », nous voyons mal au surplus qui, en dehors d'Edmond de Rothschild, aurait été en mesure de convaincre l'état-major de l'Alliance, si dévoué aux intérêts français, à s'engager dans pareille voie. Or, une lettre émanant du secrétariat de l'Alliance, autrement dit de Jacques Bigart, affirme en juin 1915, qu'il vaut mieux « au point de vue israélite que le Protectorat de Palestine [soit] exercé par l'Angleterre plutôt que par la France dont les préoccupations confessionnelles pourraient contrarier les vues et les intérêts des Juifs " ${ }^{13}$. C'est là une profession de foi en rupture complète avec l'esprit de l'institution.

\section{Instrumentaliser le sionisme}

en jouant sur les mots (fin 1915 - printemps 1917)

Du début de l'année 1915 au printemps 1917, Lucien Wolf mène donc une politique subtile, entretenant un dialogue avec les autorités politiques alliées sur des revendications «sionistes », qui n'ont qu'un rapport lointain avec celles des sionistes de l'OSM. Observée de près, son action ne se déroule pas sans tiraillements entre les partenaires pour le compte desquels il œuvre. Sans surprise, on observe en effet que l'Alliance désirerait qu'il y mette plus de retenue que ne le préconise le Baron; elle milite aussi auprès de ses partenaires pour que la revendication «sioniste» figure en bas de leur liste de priorités, loin après la question des Juifs russes. Elle a du mal aussi à se résigner, semble-t-il, à l'idée d'une tutelle britannique sur la Palestine ${ }^{14}$.

Au total, cette politique israélite imparfaitement unifiée enregistre, dans le courant de 1916, certains résultats mais qui font long feu. Le premier survient dans le cadre de la campagne de propagande que les Français, aidés de leurs notables israélites, ont décidé d'entreprendre à la fin de l'année précédente auprès des Juifs des pays neutres. Le professeur Victor Basch a accepté d'accomplir une mission aux États-Unis

\footnotetext{
12 Élisabeth Antébi, op. cit.; Jehuda Reinharz, Chaïm Weizmann. The making of a Zionist leader, New York/Oxford, Oxford University Press, 1985.

${ }^{13}$ AAIU, Secrétariat, registre S. 238 (juillet 1914-novembre 1915), lettre, 23 juin 1915.

${ }^{14}$ Mark Levene, op. cit. passim.
} 
(novembre 1915-mars 1916). Cet intellectuel socialiste, spécialiste de la philosophie allemande et germaniste distingué, éminent dreyfusard et militant de la Ligue des Droits de l'Homme, est aussi, c'est moins connu, un proche du baron Edmond de Rothschild; sa conversion soudaine et publique à un sionisme de type philanthropique en 1911 (ce qui ne saurait être un hasard) a fait du bruit à l'époque dans les milieux juifs et sionistes parisiens ${ }^{15}$. Officiellement chargé par le Quai d'Orsay de convaincre les Juifs américains que la véritable patrie de l'antisémitisme était l'Allemagne, et non pas la Russie, et que la cause alliée était donc bien la cause des Juifs, Basch a manifestement voulu partir muni d'arguments moins exclusivement rhétoriques, dans une veine où nous reconnaissons la main du Baron et de ses partenaires.

Après avoir, à son tour, échoué à obtenir une intervention de Paris auprès du gouvernement de Petrograd en faveur des Juifs russes, on le voit en effet insister auprès du ministre des Affaires étrangères, Aristide Briand, et de ses collaborateurs pour être autorisé à promettre le soutien de la France à la cause « sioniste ». L'autorisation s'est fait attendre, mais elle parvient finalement à Basch le 12 février 1916, alors qu'il se trouve à New York, via un télégramme signé Philippe Berthelot ${ }^{16}$, le directeur de cabinet de Briand: le ministre l'autorise finalement, «à titre confidentiel », à déclarer à ses interlocuteurs américains qu' « au cours des pourparlers qui ont eu lieu entre la France, l'Angleterre et la Russie au sujet de l'Asie Mineure [allusion aux négociations des accords SykesPicot, en cours], la défense, l'extension et la liberté des colonies juives de Palestine ne seront pas oubliées par la France et l'Angleterre $»$.

Et c'est, à nouveau comme par hasard, un homme très proche de l'institution, Nissim Behar, assisté de son fils, tous deux installés depuis de longues années déjà aux États-Unis, qui traduit ce texte en anglais et le diffuse (sans grand succès semble-t-il) dans la presse juive d'outreAtlantique $^{17}$. Mais le gouvernement français n'assume ni ne dément ce

\footnotetext{
${ }^{15}$ Catherine Fhima et Catherine Nicault, «Victor Basch et la judéïté », dans Françoise Basch, Liliane Crips, Pascale Gruson (dir.), Victor Basch, 1863-1944. Un intellectuel cosmopolite, Berg International Éditeurs, 2000, pp. 199-236. Le développement sur la mission de Victor Basch aux États-Unis s'inspire de cette étude.

${ }^{16}$ Archives du ministère français des Affaires étrangères, La Courneuve (désormais : AMAE-La Courneuve), Guerre 1914-1918, Sionisme 1197, télégramme signé pour ordre de Philippe Berthelot sur autorisation d'Aristide Briand et transmis à V. Basch par le consulat général de France à New York.

${ }^{17}$ Né à Jérusalem en 1848 , Nissim Béhar avait fait partie de la première promotion de l'École Normale Israélite Orientale (ENIO). Nommé directeur de l'école de Jérusalem
} 
texte que les sionistes français, Basch en tête, n'hésitèrent pourtant pas à appeler a posteriori la «Déclaration Briand ${ }^{18}$. Surtout, la promesse contenue dans le document est tellement vague qu'elle n'eut aucun impact sur les leaders du sionisme américain, déjà gagnés au demeurant à la ligne de Chaïm Weizmann. Encore ne sont-ils pas en mesure de détecter, à l'époque, les approximations et les contrevérités du texte. Car les pourparlers franco-britanniques qui se terminent sur «l'Asie mineure » concernaient avant tout, en fait, les provinces arabes de l'Empire ottoman; ils sont signés entre les Français et les Britanniques en février 1916, élargis ensuite aux Russes et aux Italiens ${ }^{19}$; et surtout, il n'est pas question des Juifs dans les accords dits «Sykes-Picot» qui prévoient pour la Palestine un «régime international», sans autres précisions.

Un mois plus tard, Lucien Wolf obtient une ouverture nettement plus prometteuse. Edward Grey, le chef du Foreign Office dans le cabinet Asquith, accueille en effet avec un certain intérêt sa proposition de publier une déclaration franco-britannique sur les Juifs de Palestine ainsi libellée :

$\mathrm{Au}$ cas où la Palestine entrerait dans les sphères d'influence de la GrandeBretagne ou de la France à la fin de la guerre, les gouvernements de ces puissances ne manqueraient pas de tenir compte de l'intérêt historique de ce pays pour la communauté juive. La population juive aurait l'assurance de jouir de la liberté civile et religieuse, de l'égalité des droits politiques avec le reste de la population, de facilités raisonnables pour l'immigration et la colonisation et de tous les privilèges municipaux dans les villes et les colonies habitées par eux qui s'avéreront nécessaires. ${ }^{20}$

en 1882, il est envoyé aux États-Unis par l'Alliance en 1901. Sa position sur le sionisme était sensiblement plus nuancée que celle des grands notables de l'Alliance.

18 Catherine Fhima et Catherine Nicault, «Victor Basch et la judéïté », op. cit. Il s'agissait ainsi de fonder la thèse, erronée, d'une primauté française dans la reconnaissance des revendications sionistes qui flattait manifestement la vanité de Victor Basch.

19 Ces accords seront complétés avec les Russes en mai 1916 et avec l'Italie en avril 1917 (accords de Saint-Jean de Maurienne), sans modification des clauses francobritanniques initiales concernant les territoires arabes de l'Empire ottoman. Textes et cartes dans Antoine Hokayem, Marie-Claude Bittar, L'Empire ottoman, les Arabes et les grandes puissances 1914-1920, Beyrouth, Les Éditions universitaires du Liban, 1981. Ces accords ont fait couler beaucoup d'encre. Citons entre autres deux études en français parmi bien d'autres : Nadine Picaudou, La Décennie qui ébranla le MoyenOrient (1914-1923), Bruxelles, Complexe, 1992 ; Vincent Cloarec, La France et la question de Syrie (1914-1918), préface d'Henry Laurens, Paris, CNRS Éditions, 2010.

${ }^{20}$ AAIU, Angleterre I J 7, courrier de L. Wolf à Georges Leygues, président du Comité d'information et d'action auprès des Juifs des pays neutres, un comité officiel animé par 
On voit bien que, loin de leur donner pleine satisfaction, cette « formule », comme on désigne désormais ce projet de déclaration franco-britannique dans les chancelleries concernées, est nettement plus susceptible de rallier les sionistes disciples alors de Weizmann que la «Déclaration Briand». La meilleure preuve est qu'informés un mois plus tard de sa démarche, Weizmann et ses amis se sont estimés trahis par Wolf, avec qui ils cultivaient jusque-là de bonnes relations ${ }^{21}$.

Las! Paris décline l'offre soumise par Grey en mars $1916^{22}$. Sans doute les milieux catholiques, désignés comme responsables de ce refus par Wolf et ses commanditaires, et, ajouterons-nous, le « parti syrien » de Paris, groupe informel de diplomates, d'hommes d'affaires, de militaires, de religieux anxieux que revienne à la France après la guerre une «Grande Syrie» comprenant la Terre sainte ${ }^{23}$, eurent-ils une part essentielle dans ce refus. Mais il nous est difficile de croire que l'Alliance soit étrangère à cette décision, tant elle se montre par ailleurs hostile à toute idée de "privilèges » octroyées aux Juifs, que ce soit en Palestine ou ailleurs.

Somme toute, le programme «sioniste» de la «diplomatie israélite » n'a pas remporté de francs succès dans les premiers mois de 1916. Mais les premiers contacts noués alors par Weizmann avec des membres du gouvernement britannique ne sont pas plus décisifs. À cette époque, avant l'entrée en guerre des États-Unis aux côtés de l'Entente (avril 1917), les choses sérieuses se déroulent au niveau diplomatique les alliés français et britanniques négocient les accords secrets dits SykesPicot qui décident des lignes du partage futur des territoires arabes de

diverses personnalités israélites, la plupart liées de près ou de loin à l'Alliance, 20 mars 1916 (il s'agit d'une traduction de l'anglais réalisée par Jacques Bigart lui-même).

${ }^{21}$ Mark Levene, op. cit., pp. 99 et 114 . Pour cet historien, ladite « formule » aurait été conçue par Lucien Wolf sans qu'il ait consulté les sionistes mais dans un souci sincère de trouver un compromis avec eux.

22 AMAE-La Courneuve, Guerre 1914-1918, Turquie 872, note de Grey au Quai d'Orsay, 12 mars 1916. Document publié par Isaiah Friedman, dans The Rise of Israel, vol 5 : France and Zionism, 1914-1920, Garland Pub. Inc., New York et Londres, 1987.

${ }^{23}$ Sur le «parti syrien », fraction du parti colonial en France : C.M. Andrews et A.S. Kanya-Fortsner, The Climax of French Expansion, Stanford University Press, Stanford, Californie, 1981 ; idem, " La France et la recherche de la "Syrie intégrale" 1914-1920 », Relations internationales, 19, automne 1979, pp. 263-278; et plus récemment les travaux de Julie d'Andurain, dont « La Méditerranée orientale, nouvel enjeu entre la France et la Grande-Bretagne durant la Grande Guerre », Cahiers de la Méditerranée, $\mathrm{n}^{\circ} 81$, décembre 2010, pp. 25-44, et Le Parti colonial en pensée et en action, Paris, Hémisphères éditions Zellige, 2017. 
l'Empire ottoman - et au niveau militaire : si, pour l'heure, les tentatives de l'armée britannique pour conquérir la Palestine ottomane à partir de l'Égypte ont avorté, il est clair que c'est partie remise pour Londres et que, selon toute probabilité, les Français, incapables de distraire les troupes nécessaires du front occidental, ne seront pas ou peu en mesure de participer à une future offensive, susceptible pourtant de remettre en cause les accords signés.

\section{La diplomatie israélite prise à son propre piège (1917-1918)}

Or, la fenêtre d'opportunité dont, toutes proportions gardées, la diplomatie israélite avait disposé tend à se refermer au tournant des années 1916 et 1917 avec l'arrivée au pouvoir de Lloyd George à Londres (décembre 1916) et l'importance grandissante, après l'entrée en guerre des États-Unis, du facteur des « nationalités » dans les discussions alliées relatives à l'après-guerre. À la différence de ses prédécesseurs libéraux, le nouveau Premier ministre britannique, soutenu par les milieux impérialistes, est en effet déterminé à établir une domination anglaise en Palestine, ne serait-ce que pour garantir la sécurité du canal de Suez, et s'emploie à obtenir dans ce but le soutien des diverses « nationalités » de la région. C'est au tour de Weizmann et de ses amis de disposer d'une fenêtre d'opportunité dont ils firent bon usage: les négociations discrètes engagées avec le Foreign Office en février 1917 aboutirent à la Déclaration Balfour, le 2 novembre suivant.

L'Alliance et ses partenaires sont à mille lieux de se douter, au début de 1917, que le nouveau contexte a résolu la poignée de diplomates en charge des affaires d'Orient au Quai d'Orsay à opérer une volte-face. François Georges-Picot notamment, le négociateur français des accords éponymes, imagine en effet, plusieurs mois avant ses collègues britanniques, que les chances de voir appliquer les accords Sykes-Picot seraient meilleures s'il pouvait se targuer du soutien des sionistes, qu'il tient ou affecte de tenir, pour les porte-parole de la « nationalité » juive ; lui et ses collègues du Quai espèrent encore, en effet, obtenir l'établissement d'un futur condominium franco-britannique sur une Palestine toujours ottomane à cette date. Représentant le Comité politique sioniste, Nahum Sokolow, un proche de Chaïm Weizmann tenu à tort par le Quai d'Orsay pour être l'homme fort du mouvement, est donc invité à Paris au début de 1917. Au courant bien entendu des pourparlers secrets engagés avec le Foreign Office par Weizmann, décidé 
à jouer exclusivement la carte britannique, il a la surprise de se voir offrir un marché pour le moins inattendu: une déclaration française de sympathie pour les aspirations sionistes en échange d'un soutien tacite des sionistes pour la préservation, au moins partielle, après la guerre des intérêts français en Palestine ${ }^{24}$. Bravant le mécontentement de Weizmann, il accepte la publication, le 4 juin 1917, de ce qu'il faut, à juste titre cette fois, appeler la «Déclaration Cambon», du nom du secrétaire général du Quai, Jules Cambon, signataire du texte :

\begin{abstract}
Vous avez bien voulu m'exposer le projet [...] de développer la colonisation israélite en Palestine. Vous estimez que si les circonstances le permettent et l'indépendance des Lieux saints étant assurée d'autre part, ce serait faire œuvre de justice et de réparation que d'aider à la renaissance, par la protection des puissances alliées, de la nationalité juive, sur cette terre d'où le peuple juif a été chassé il y a tant de siècles.

Le gouvernement français qui est entré dans la présente guerre pour défendre un peuple injustement attaqué et qui poursuit la lutte pour assurer le triomphe du droit sur la force, ne peut éprouver que de la sympathie pour votre cause dont le triomphe est lié à celui des Alliés.

Je suis heureux de vous en donner ici l'assurance. ${ }^{25}$
\end{abstract}

Les raisons pour lesquelles la Déclaration Cambon est resté lettre morte tant pour les sionistes que pour les autorités françaises ne nous intéressent pas $\mathrm{ici}^{26}$. En revanche, il importe de souligner pour notre propos que le séjour de Sokolow du printemps 1917 à Paris fut un choc sévère pour l'état-major de l'Alliance. Sokolow a voulu profiter de son séjour parisien pour obtenir - aisément croyait-il - le ralliement des notables du Consistoire central et de l'Alliance au mouvement qu'il représentait et que le gouvernement français s'apprêtait à reconnaître. Par le journal intime tenu par Sokolow, nous sommes pour le coup assurés de

\footnotetext{
${ }^{24}$ Sur cet épisode : outre M. Weisgal (ed.), The Letters and Papers of Chaïm Weizmann, Series A Letters, Londres et Jérusalem, 1968-1985, vol. VII ; André Kaspi (dir.), chapitre 5, op. cit., et Catherine Nicault, La France et le sionisme..., op. cit.

${ }^{25}$ AMAE-La Courneuve, guerre 1914-1918, 1199 ; Central Zionist Archives, Jérusalem (désormais : CZA), A18/24. Document reproduit dans le recueil d'Isaiah Friedman (ed.), op. cit. Les intentions de Sokolow mériteraient d'être éclaircies: souci de contrebalancer le leadership montant de Weizmann dans le mouvement sioniste ? De ne pas alerter trop tôt les Français en rejetant leur proposition ou même de faire pression sur le Foreign Office, encore très partagé sur l'opportunité de publier une déclaration pro-sioniste et surtout sur son contenu?

${ }^{26}$ À ce sujet: C. Nicault, La France et le sionisme..., op. cit., pp. 76-82; Henry Laurens, La Question de la Palestine, I : 1799-1922, L'invention de la Terre sainte, Paris, Fayard, 1999.
} 
l'implication d'Edmond de Rothschild, qui semble jouer désormais Sokolow contre Weizmann pour l'accomplissement de son grand dessein (réunir derrière lui toutes les forces vives du judaïsme), dans toute cette affaire. C'est en effet par Sokolow, venu en personne expliquer au Comité central de l'Alliance ses tractations avec les autorités françaises, que ses membres apprennent, le 14 avril 1917, qu'ils sont en somme «lâchés» par leur propre gouvernement. C'est pour la plupart la consternation, l'incompréhension et le désarroi. Leur collègue, Sylvain Lévi, un savant indianiste de premier plan, très proche d'Edmond de Rothschild, prend manifestement le parti, sous l'influence évidente du Baron qui persiste à penser que tout n'est pas perdu, de se plier aux desiderata du Quai tout en travaillant pour qu'on y entende le sionisme à sa façon, et contrer ainsi les projets politiques weizmanniens dans ce qu'ils ont, à leurs yeux, d'éminemment dangereux ${ }^{27}$.

En tout cas, lorsque la Déclaration Balfour est publiée en novembre 1917, alors que le général Allenby entame, pour de bon cette fois, la conquête de la Palestine, la diplomatie française persiste dans l'option adoptée au début de l'année: elle confirme sa sympathie pour le sionisme et apporte son soutien à la promesse britannique de favoriser la création d'un foyer national juif en Palestine. Si, à Paris, l'on a fait son deuil à cette date d'un condominium franco-britannique en Palestine, l'on espère encore ménager l'établissement d'un régime international plus large au sein duquel la France aurait son rôle à jouer. C'est pourquoi le sionisme ne saurait être abandonné aux seuls Britanniques, même s'il faudrait être aveugle et sourd pour ignorer la préférence marquée des sionistes pour la Grande-Bretagne. D'où une seconde invitation à Sokolow à Paris et la publication de la Déclaration Pichon, du nom du ministre des Affaires étrangères de Clemenceau, le 14 février 1918 :

Monsieur,

Comme il a été convenu au cours de notre entretien le samedi 9 de ce mois, le gouvernement de la République, en vue de préciser son attitude vis-à-vis des aspirations sionistes, tendant à créer pour les Juifs en Palestine un Foyer national, a publié un communiqué dans la presse [paru le 9 février 1918].

En vous communiquant ce texte, je saisis avec empressement l'occasion de vous féliciter du généreux dévouement avec lequel vous poursuivez la réalisation des vœux de vos coreligionnaires, et de vous remercier du zèle que vous apportez à

\footnotetext{
${ }^{27}$ Sur le premier séjour de Sokolow à Paris : C. Nicault, « Face au sionisme », pp. 204207.
} 
leur faire connaître les sentiments de sympathie que leurs efforts éveillent dans les pays de l'Entente et notamment en France. [.... ${ }^{28}$

\section{Les déboires de Sylvain Lévi et l'ancrage de l'Alliance dans l'antisionisme pendant l'entre-deux-guerres}

En 1918, nous trouvons Sylvain Lévi étroitement mêlé aux relations des plus ambiguës qu'entretiennent la France et le mouvement sioniste. Très probablement introduit par le baron Edmond de Rothschild auprès des diplomates en charge des affaires d'Orient au Quai, il est pour le ministère français des Affaires étrangères l'agent des dernières tentatives, officielles et officieuses, pour contrer les ambitions sionistes tout en prétendant les soutenir. Après deux missions à l'étranger, il est discrètement promu au Quai, à l'approche de l'ouverture de la Conférence de la Paix, conseiller pour les affaires juives. Ce ne fut qu'une suite d'échecs qui, consommant la défaite de la diplomatie israélite alliée, achevèrent de braquer la direction de l'Alliance contre les sionistes et singulièrement contre l'homme destiné à diriger l'Organisation sioniste mondiale après la guerre, Chaïm Weizmann. Trois épisodes principaux jalonnent cette déroute.

En mars 1918, pour commencer, le Foreign Office, d'accord avec Weizmann, décide de faire venir en Palestine, encore incomplètement conquise par les Anglais, une Commission sioniste chargée, en théorie, d'y enquêter sur la situation des Juifs sur place, en réalité pour y préparer la mise en œuvre de la Déclaration Balfour. Quelque désir qu'en aient Londres et Weizmann, il leur est impossible d'en exclure la France, puisqu'en théorie l'occupation de la Palestine par les Britanniques ne préjuge en rien les décisions de la future Conférence de la Paix. Le Baron et la diplomatie française en profitent pour imposer l'adjonction de Sylvain Lévi à ladite Commission. Parti en Palestine, il se sentit sans prise aucune sur les événements, de plus en plus mal à l'aise au spectacle d'un sionisme weizmannien en action où il décèle, entre autres, une influence bolchevique qui l'horrifie, prenant totalement en grippe

\footnotetext{
${ }^{28}$ La Déclaration Pichon figure dans AMAE-La Courneuve, Guerre 1914-1918, 1200. Sur le second séjour de Sokolow à Paris : ibid., pp. 89-94. On doit à Yoram Majorek une traduction en hébreu du journal intime de Sokolow, en polonais, (Nahum Sokolov's Paris Diary, January-february 1918, Jérusalem, Université hébraïque, juin 1988, 104 p.), texte à son tour traduit en français par Rachel Garbarz et Jean-Marie Delmaire dans une version abrégée: Yoram Majorek, «Le journal de Nahum Sokolow à Paris (janvier-février 1918) », Tsafon. Revue d'études juives du Nord, n 11-12, printemps-été 1993, pp. 3-55.
} 
Weizmann, le président de la Commission sioniste, qui le lui rend bien. Outre cette hostilité réciproque désormais déclarée, le résultat le plus clair de ce séjour est d'avoir encore rapproché, s'il en était besoin, Sylvain Lévi de l'Alliance dont il a admiré les écoles palestiniennes ${ }^{29}$.

Il y a gagné également la pleine confiance du Quai qui lui confie, très vite après son retour de Palestine, une autre mission, aux États-Unis cette fois, qu'il accomplit de septembre à décembre 1918. Il s'agit de rallier les sionistes américains, tenus pour plus raisonnables que les sionistes weizmanniens, au projet d'une conférence qui se tiendrait entre représentants du judaïsme de Grande-Bretagne, de France et des ÉtatsUnis, laquelle favoriserait la fondation en Palestine d'une sorte de compagnie à charte « qui, tout en assurant les colonisations des Sionistes et en leur donnant la plus large autonomie possible, prévienne les dangers qui pourraient naître des excès de l'esprit nationaliste de certains sionistes $\gg{ }^{30}$. Ce plan, qui ne peut qu'être sorti du cerveau du Baron, déboucha sur un fiasco humiliant pour Sylvain Lévi. Mal reçu aux ÉtatsUnis, il en revint très remonté contre les leaders du sionisme américain et contre le sionisme qu'il dépeint désormais comme «une mode passagère » permettant aux millionnaires juifs américains de «s'assur[er] contre un surplus d'immigration juive », sans réaliser qu'ils développent ainsi en Palestine « un centre d'influence et d'action russo-germanique », autrement dit bolchevique ${ }^{31}$.

La rupture, déjà consommée dans son esprit, l'est publiquement à l'occasion de la Conférence de la Paix, précisément lors de la fameuse comparution de la délégation sioniste devant le Conseil des Dix, le 27 février 1919. Sur l'intervention insistante du Quai, toujours conseillé par Edmond de Rothschild, Sylvain Lévi fait partie de cette délégation que conduit Weizmann au titre d'un fantomatique « sionisme français » dont la diplomatie française persiste à entretenir la quasi-fiction. Pour contrebalancer la présence de Lévi, que les sionistes présument peu sympathique à leur cause, Sokolow est parvenu à faire nommer en dernière minute André Spire comme le second représentant du « sionisme français »- avec plus de raisons, en l'occurrence, puisque ce dernier est le fondateur à Paris d'une Ligue des amis français du sionisme

\footnotetext{
${ }^{29}$ C. Nicault, « Face au sionisme », pp. 212-215.

${ }^{30}$ AMAE, E Levant, Sionisme, 10, note de Jean Goût pour le ministre, 22 juillet 1918, avec cette annotation manuscrite : «approuvé S. P. [Stephen Pichon]. J'ai vu le baron de Rothschild ».

${ }^{31}$ AMAE, E Levant, Sionisme, 11, notes de voyage de Sylvain Lévi, entrées du 23 et 29 septembre, du 5 octobre et du 3 novembre 1918.
} 
qui collabore activement avec la délégation sioniste. Toujours est-il que, comme Weizmann et ses amis le pressentaient, Lévi fit une longue déclaration fondée sur un distinguo entre un «bon » sionisme, visant la seule mise en valeur de la Palestine, et le « mauvais » sionisme, celui que professent les autres membres de la délégation. Pour ces derniers, pénétrés de la solennité du moment et de la gravité de l'enjeu, c'en est trop : Lévi est plus un traître à la cause juive qui ne mérite que colère, sarcasmes et mépris ${ }^{32}$.

Du coup, l'antagonisme entre l'Alliance et le mouvement sioniste s'en est trouvé exacerbé. De fait, l'élection de Sylvain Lévi à la présidence de l'Alliance par ses pairs du Comité central le 27 juin 1920 doit être interprétée, selon nous, comme une réponse cinglante de l'institution au sionisme weizmannien et un manifeste marquant la ferme intention de l'institution de camper résolument dorénavant sur ses principes $^{33}$. Cela, au risque de contrarier les autorités politiques françaises dont le bras de fer avec Londres s'est entretemps déplacé, au fond, sur la seule Syrie, mais qui persiste à se servir de la Palestine comme d'un argument de marchandage. En mai 1918 déjà, le comité central avait refusé tout net de faire preuve, à leur demande, de plus de souplesse vis-à-vis du sionisme ${ }^{34}$. Elle réitère indirectement ce refus par sa déclaration du 20 décembre 1922 affirmant une «neutralité » à l'égard du sionisme qui ne trompe personne. Il y a beau temps, dit le texte, que :

[L'Alliance a fait] preuve de l'intérêt particulier qu'elle porte à la Palestine et à tout ce qui peut contribuer au relèvement économique et moral de la population juive de ce pays. Mais de même qu'elle s'est toujours tenue en dehors des dissidences religieuses qui se sont produites au sein du judaïsme, l'Alliance ne saurait prendre parti dans les problèmes concernant le sionisme, qui soulèvent des problèmes d'ordre politique, et sur lesquels l'accord est loin d'être réalisé,

\footnotetext{
32 C. Nicault, La France et le sionisme..., op. cit., pp. 118-119; André Spire, « Du Mahayàna Sutràlankara à la conférence de la paix (essai de contribution à l'éclaircissement du cas Sylvain Lévi) », La Palestine nouvelle, mars 1918 (avec la participation de Samuel Tolkowsky), repris dans idem, Souvenirs à bâtons rompus, Paris, Albin Michel, 1962.

${ }^{33}$ Le communiqué publié par le nouvel élu est cité dans André Chouraqui, L'Alliance israélite universelle et la renaissance juive contemporaine. Cent ans d'histoire, Paris, Puf, 1965, p. 486.

${ }^{34}$ CZA, A93/6, «Allocution de M. Georges-Picot au consistoire israélite de France, 27 août 1918 », Le Peuple juif, déclaration au Comité d'études sionistes, le 29 août 1918, $\mathrm{n}^{\circ} 4$, juillet 1919 .
} 
entre les israélites eux-mêmes. Elle se doit en conséquence de persévérer dans l'attitude de neutralité qu'elle a observée jusqu'à ce jour. ${ }^{35}$

Le gouvernement français se le tint pour dit, usant au besoin d'autres canaux pour entretenir des rapports corrects, quoique prudents, avec un mouvement sioniste investi de responsabilités importantes dans la Palestine voisine des États français du Levant. Dès lors, si le Baron semble s'être accommodé de la situation, voyageant à plusieurs reprises en Palestine mandataire jusqu'à son décès en 1934, l'Alliance s'enferme dans une attitude d'opposition rigide au sionisme et à ses représentants. Celle-ci contribue à l'isoler dans le monde juif de l'entre-deux-guerres, tout particulièrement dans les années trente, lorsque le danger antisémite en l'Europe centrale et orientale persuade bon nombre de notables communautaires de l'intérêt de disposer pour les abriter d'un refuge éventuel dans le Foyer national juif.

\footnotetext{
${ }^{35}$ AAIU, France, I G 4. Le comité central a pris la décision de faire cette déclaration le 25 novembre 1922, selon AAIU, Syrie I G 1, lettre de Rosenfeld, médecin à Alep, à Bigart, 20 décembre 1922.
} 